\title{
Propiedades psicométricas del Hopkins Symptom Checklist (HSCL-11) en Argentina: Un instrumento para monitoreo y feedback en psicoterapia
}

\author{
Psychometric Properties of the Hopkins Symptom Checklist (HSCL-11) in Argentina: An \\ instrument for monitoring and feedback in Psychotherapy
}

\author{
Juan Martín Gómez-Penedo ${ }^{* 1,2}$, Malenka Areas ${ }^{1,2}$, Rocío Manubens ${ }^{1}$, Anna Babl ${ }^{4}$, Laura Challú ${ }^{3}$, Santiago Juan ${ }^{1}$, \\ Roberto Muiños ${ }^{1}$, Javier Fernández-Álvarez ${ }^{5,6}$, Nicolás Alalú ${ }^{1}$, Andrés Roussos ${ }^{1,2}$, Wolfgang Lutz $^{7}$, \\ Martin grosse-Holtforth ${ }^{4,8}$ \\ 1 - Universidad de Buenos Aires, Buenos Aires, Argentina. 2 - CONICET, Buenos Aires, Argentina. \\ 3 - Universidad de Belgrano, Buenos Aires, Argentina. 4 - Universität Bern, Berna, Suiza. \\ 5 - Fundación Aiglé, Buenos Aires, Argentina. 6 - Universitat Jaume I, Castellón de la Plana, España. \\ 7 - Universitäty of Trier, Tréveris, Alemania. 8 - Universitätsspital Insel, Berna, Suiza. \\ Introducción \\ Método \\ Resultados \\ Discusión \\ Referencias
}

Recibido: 23/10/2020 Revisado: 22/12/2020 Aceptado: 11/01/2021

\section{Resumen}

No se han encontrado instrumentos breves válidos en Argentina para monitorear sintomatología psicológica en psicoterapia. Este trabajo presenta la adaptación argentina del Hopkins Symptoms Checklist (HSCL-11) y estudia sus propiedades psicométricas. Una muestra de 356 participantes completó la adaptación del HSCL-11 junto con medidas de depresión, ansiedad y bienestar. Se computaron el coeficiente alfa de Cronbach y correlaciones ítem-total corregidas. Se realizó un análisis factorial confirmatorio, se estudiaron sus correlaciones con las otras medidas y se analizaron las diferencias en HSCL-11 entre pacientes y no pacientes de terapia. Se observaron evidencias de adecuada consistencia interna y homogeneidad de ítems, así como también de validez de constructo, validez concurrente y validez de criterio. Los resultados mostraron evidencias de confiabilidad y validez de la versión argentina del HSCL-11, sugiriendo que podría representar un instrumento valioso para la evaluación y el tratamiento de patologías mentales en el país.

Palabras clave: Hopkins Symptom Checklist versión argentina, propiedades psicométricas, validez, confiabilidad, sintomatología psiquiátrica, ansiedad, depresión, monitoreo de psicoterapias

\begin{abstract}
We failed to find brief instruments validated in Argentina to explore and monitor psychological symptoms from psychotherapy patients. This paper presents the Argentinian adaptation of the Hopkins Symptoms Checklist (HSCL-11) and analyzes its psychometric properties. A sample of 356 participants completed the HSCL-11 adaptation, along with other measures of depression, anxiety, and well-being. We analyzed Cronbach's alpha and item-total correlations adjusted. We ran confirmatory factor analysis, correlations among the measures, and examined differences in HSCL-11 between subjects who were psychotherapy patients and those who were not. We found evidence of adequate internal consistency and item homogeneity, as well as construct validity, concurrent validity, and criterion validity. Results showed evidence of reliability and validity for the HSCL-11 Argentinian adaptation, suggesting that it might represent a meaningful resource for the evaluation and treatment of mental disorders in Argentina.
\end{abstract}

Keywords: Hopkins Symptom Check-Lists Argentine version, psychometric properties, validity, reliability, psychiatric symptomatology, anxiety, depression, psychotherapy monitoring

\footnotetext{
* Correspondencia a: Juan Martín Gómez Penedo. Lavalle 2353, Buenos Aires, Argentina. Teléfono: +54 9116525 7053. E-mail: jmgomezpenedo@gmail.com Cómo citar este artículo: Gómez-Penedo, J. M., Areas, M., Manubens, R., Babl, A., Challú, L., Juan, S., ... grosse-Holtforth, M. (2021). Propiedades psicométricas del Hopkins Symptom Check-Lists (HSCL-11) en Argentina: Un instrumento para monitoreo y feedback en psicoterapia. Revista Evaluar, 21(2), 33-47. Recuperado de https://revistas.unc. edu.ar/index.php/revaluar

Nota de autor: Este proyecto fue financiado con un 2020 Seed Money Grant, otorgado por la Leading House for the Latin American Region del Centro Latinoamericano-Suizo de la Universität St. Gallen (Suiza). Los Investigadores principales del proyecto fueron Dr. Juan Martín Gómez Penedo y Dr. Martin grosse Holtforth. Participaron en la edición de este artículo: Julian Narvaja, Carolina Liendo, Eugenia Maiorana, Alicia Molinari, Juan Cruz Balverdi, Florencia Ruiz, Ricardo Hernández.
} 


\section{Introducción}

En los últimos años se ha observado en el campo de la psicoterapia un interés creciente por el monitoreo regular de los resultados de los tratamientos y por la utilización de esta información para proveer feedback a los terapeutas (Boswell, Constantino, Kraus, Bugatti, \& Oswald, 2016; Emmelkamp et al., 2014; Lutz, Rubel, Schwartz, Schilling, \& Deisenhofer, 2019). Recabar dicha información y transmitirla a los terapeutas en tiempo real permite tener un seguimiento de la evolución terapéutica de cada paciente, identificando, por ejemplo: (i) casos que han mejorado significativamente a partir de la terapia, (ii) casos que han presentado una respuesta menor a la esperada y (iii) casos que se han deteriorado, empeorando su condición clínica a partir del tratamiento (Lambert, 2013). Además de evaluar la evolución de los pacientes, estos instrumentos de seguimiento podrían permitir identificar marcadores de riesgo en vivo que deberían ser inmediatamente abordados, como es el caso de la ideación suicida (Lutz et al., 2019). Al contar con esta información, los terapeutas pueden implementar intervenciones específicas y adaptaciones de los tratamientos en función de las necesidades específicas de los pacientes, orientadas a optimizar los abordajes y sus resultados (Constantino, Coyne, \& Muir, 2020).

Para poder realizar un seguimiento regular de la evolución clínica de los pacientes es necesario contar con instrumentos que sean robustos psicométricamente. Al mismo tiempo, es importante que sean instrumentos sencillos y rápidos de completar, de modo que reduzcan la carga de tiempo y esfuerzo sobre los pacientes, y permitan su administración de forma rutinaria (por ejemplo, sesión a sesión).

Existen diferentes instrumentos que permiten medir sintomatología psicológica y resultados en psicoterapia. En Iberoamérica algunos de ellos han sido traducidos al español y validados localmente, mostrando adecuadas propiedades psicométricas. Uno de los ejemplos más difundidos es el del Outcome Questionnaire (OQ-45; Lambert et al., 1996; Lara, Cruz, Vacarezza, Florenzano, \& Trapp, 2008), cuyo uso en países como Chile y Argentina es frecuente (p. ej., Barros et al., 2020; Gómez-Penedo, Zilcha-Mano, \& Roussos, 2019; Roussos, Gómez-Penedo, \& Muiños, 2018; Zilcha-Mano \& Errázuriz, 2015). Dicho cuestionario se presenta como una medida psicométricamente rigurosa, que abarca los motivos de consulta más comunes de las personas que solicitan psicoterapia, y que es sensible a modificaciones en períodos breves, lo que facilita su uso para monitorear procesos de cambio. Otro de los instrumentos utilizados con validaciones en España, Ecuador y México es el Cuestionario de Evaluación de Resultados CORE Outcome Measure (CORE-OM; Botella i García del Cid, 2008; Feixas et al., 2012; Paz, Mascialino, \& Evans, 2020; Sosa-Torralba, Romero-Mendoza, Medina-Mora-Icaza, Méndez-Ríos, \& Blum-Grynberg, 2020). Dicho cuestionario consta de 34 ítems autoadministrados que evalúan un amplio conjunto de síntomas de malestar psicológico en cuatro dimensiones: bienestar subjetivo, síntomas, funcionamiento y riesgo. Otra de las opciones es el Listado de Síntomas Breve (LSB-50), instrumento de evaluación psicopatológica utilizado para la detección de síntomas psicológicos y psicosomáticos (Abuín \& de Rivera, 2014). El mismo está formado por 50 ítems descriptivos de manifestaciones sintomáticas psicológicas y psicosomáticas.

Específicamente en Argentina, se cuenta con un conjunto de instrumentos validados que permiten medir sintomatología y evolución de los pacientes a lo largo del tratamiento, como es el caso del ya mencionado OQ-45 (Fernández-Álvarez, Hirsch, Maristany, \& Torrente, 2005). Por 
otra parte, también se ha realizado una adaptación y validación del Symptom Checklist - 90 (SCL90; Casullo \& Pérez, 1999/2008). El SCL-90 es un instrumento que evalúa, en 90 ítems, 9 dimensiones diferentes de sintomatología psiquiátrica y 3 índices de malestar psicológico. Las escalas sintomáticas incluyen: Somatización, Obsesión-compulsión, Sensibilidad interpersonal, Depresión, Ansiedad, Hostilidad, Ansiedad fóbica, Ideación paranoide y Psicoticismo. Los índices de malestar son: a) el índice global de severidad (GSI), b) el índice de malestar sintomático positivo (PSDI) y c) el total de síntomas positivos (PST). A su vez, en Argentina se ha adaptado el LSB-50 para población adolescente (De la Iglesia, Stover, Castro-Solano, \& Fernández-Liporace, 2015). Además de estas medidas genéricas de psicopatología, también se han adaptado en el país instrumentos para medir sintomatología asociada a cuadros diagnósticos específicos, como el Patient Health Questionnaire-9 (PHQ-9), que consiste en una breve herramienta de 9 ítems para evaluar la presencia de síntomas depresivos y su severidad (Urtasun et al., 2019).

A pesar de que en el país existen algunos instrumentos con propiedades psicométricas robustas, instrumentos como los mencionados OQ45 y SCL-90 se vuelven de difícil implementación para el monitoreo rutinario y feedback consecuente a los terapeutas en contextos naturalísticos. Fundamentalmente, esto se debe a que, al contar con una gran cantidad de ítems, estas medidas pueden representar una carga muy grande para los pacientes si se les solicita completarlos antes o después de cada sesión durante todo un tratamiento. Otros instrumentos que cuentan con menor cantidad de ítems, como el PHQ-9, están asociados a patologías específicas, lo cual limita su posibilidad de aplicación para la evaluación de pacientes con diversidad de condiciones psicopatológicas en ámbitos naturalísticos.
Recientemente se ha presentado un dispositivo integrado por tres ítems que evalúa en forma genérica la evolución de los pacientes. Cada ítem mide en forma separada los niveles de sintomatología, funcionamiento social y relaciones interpersonales de los pacientes (Areas et al., 2018). Sin embargo, a pesar de la sencillez del instrumento, por su naturaleza no ha sido posible poner a prueba sus propiedades psicométricas.

El estado del arte muestra la necesidad de contar en el contexto argentino con un instrumento de evaluación de resultados que permita monitorear sintomatología durante los tratamientos, que sea sencillo y ameno de completar para los pacientes, y que presente adecuadas propiedades psicométricas de confiabilidad y validez. De esta manera, el presente trabajo tiene por objetivo adaptar al contexto argentino el Hopkins Symptoms Checklist (HSCL-11; Lutz, Tholen, Schürch, \& Berking, 2006) y explorar sus propiedades psicométricas en términos de confiabilidad (consistencia interna y homogeneidad de ítems) y validez (validez de constructo, validez concurrente, validez de criterio).

El HSCL-11 es un instrumento de 11 ítems que evalúa sintomatología, principalmente síntomas de ansiedad y depresión, los más prevalentes en la psicopatología (Chisholm et al., 2016). Debido a sus cualidades, este instrumento ha sido previamente utilizado como recurso para monitoreo de resultados y feedback a terapeutas (Lutz et al., 2019).

Contar con un instrumento de esta naturaleza en el contexto argentino puede favorecer al campo de la psicoterapia, aportando información relevante sobre la evolución de los pacientes. De esta manera, se ofrece a los terapeutas la posibilidad de personalizar los tratamientos y por lo tanto de optimizar los resultados clínicos (Lutz, De Jong, \& Rubel, 2015). Asimismo, el monitoreo de pacientes en contextos naturales constituye 
un excelente ejemplo de investigación orientada a la práctica (Fernández-Álvarez, Fernández-Álvarez, \& Castonguay, 2018); es decir, la colaboración activa entre investigadores y clínicos trabajando en contextos rutinarios, que pueda ser significativa tanto para clínicos como investigadores. En este sentido, la adaptación al contexto argentino del HSCL-11 permitiría a los clínicos la posibilidad de tomar decisiones en función del monitoreo de sus pacientes. Los investigadores, por su parte, podrían contar con grandes volúmenes de datos sesión a sesión que, vinculados con la información de los pacientes al inicio del tratamiento, permitirían desarrollar algoritmos de resultados diferenciales para personalización y precisión en salud mental (Delgadillo \& Lutz, 2020).

\section{Metodología}

Participantes

La muestra del estudio estuvo integrada por 356 participantes. Se empleó un muestreo no probabilístico para la convocatoria de los mismos, con una estrategia de bola de nieve, mediante contactos directos y también a través del uso de diversas redes sociales. De los 311 participantes que indicaron su género, el $60.77 \%$ eran mujeres $y$ el $39.23 \%$ restante eran hombres; 45 personas (12.64\% del total) no indicaron su género. El rango de edades osciló entre los 18 y los 77 años, con una edad promedio de 36.52 años (desvío estándar $[\mathrm{DE}]=13.93)$. El $90.88 \%$ de la muestra residía en el Área Metropolitana de Buenos Aires, mientras que el $9.12 \%$ restante vivía en otras provincias de Argentina. E1 52.41\% de los participantes estaba casado, el 39.23\% sin pareja. Al preguntar sobre el máximo nivel educativo alcanzado, un 91.29\% de la muestra refirió tener estudios superiores (terciarios/universitarios) completos o incompletos. Por otra parte, el $44.05 \%$ de la muestra había efectuado tratamiento psicológico/psiquiátrico en el último año, indicando como motivos de consulta mejorar algún aspecto de su vida (48.90\%), malestar psicológico intenso $(28.47 \%)$, crisis vital $(10.95 \%)$ y otros $(11.68 \%)$.

\section{Instrumentos}

Hopkins Symptom Checklist-11 (HSCL-11, Lutz et al., 2006). Versión modificada de 11 ítems del Inventario de Síntomas-90-R (Derogatis, 1983). En el instrumento se le solicita al participante que consigne cuánto sufrió, en los últimos siete días, de un conjunto de manifestaciones psicopatológicas, en su mayoría síntomas de naturaleza ansiosa o depresiva. Los reactivos se responden en una escala Likert de cuatro puntos que oscila entre 1 (Nada) y 4 (Mucho). Algunos de los síntomas explorados son miedos, tristeza, nerviosismo y sentirse solo (ver Tabla 2 para su presentación exhaustiva). El HSCL-11 original (en alemán) mostró propiedades psicométricas adecuadas. En el estudio de Lutz et al. (2006) se observaron evidencias de consistencia interna $(\alpha$ de Cronbach $=$ $.85)$, validez de constructo y validez concurrente (Lutz et al., 2006).

Patient Health Questionnaire (PHQ-9; Kroenke, Spitzer, \& Williams, 2001) /Versión en castellano; Urtasun et al., 2019]. Esta escala de 9 ítems de autoinforme mide sintomatología depresiva de acuerdo a los criterios diagnósticos de depresión del DSM-IV. Los puntajes más altos indican mayor severidad de la sintomatología. Cada ítem se puntúa en una escala Likert que oscila entre 0 (Para nada) y 3 (Casi todos los días). Algunos ejemplos de ítems son: Se ha sentido decaído(a), deprimido(a), o sin esperanzas y Se ha sentido cansado(a) o con poca energía. En su validación argentina se observaron evidencias de alta validez 
de criterio y consistencia interna (alfa de Cronbach $=.87$; Urtasun et al., 2019).

Generalized Anxiety Disorder (GAD-7; Spitzer, Kroenke, Williams, \& Löwe, 2006) /Versión en castellano; García-Campayo et al., 2010]. Este instrumento mide sintomatología ansiosa. Consta de 7 preguntas de autoinforme que se puntúan en una escala Likert entre 0 (Nunca) y 3 (Casi todos los días). Los puntajes más altos indican mayor severidad sintomatológica. Entre los ítems del instrumento se incluyen, por ejemplo: No ha podido dejar de preocuparse y Ha tenido dificultad para relajarse. La versión española de este instrumento presenta excelentes propiedades psicométricas en su consistencia interna (alfa de Cronbach $=.93$ ), al igual que la validez discriminante y de criterio (García-Campayo et al., 2010).

\section{Well Being Index (WHO-5; Organización Mun-} dial de la Salud, 1998) (Versión en castellano; Lucas-Carrasco, 2012]. Este instrumento de autoinforme mide el bienestar subjetivo. Consiste en 5 ítems cuyas respuestas se distribuyen en una escala Likert de 6 puntos, en donde el puntaje 0 representa Nunca y el 5 representa Todo el tiempo. Puntajes altos indican mayores niveles de bienestar. El instrumento incluye ítems como: $M e$ he sentido alegre y de buen humor y Me he sentido activo y energético. En su versión argentina se hallaron adecuadas propiedades psicométricas con respecto a su consistencia interna (alfa de Cronbach $=.80)$, validez de constructo y fiabilidad (Cornelio \& Contreras, 2020).

\section{Procedimientos}

En primera instancia, para la adaptación del HSCL-11 al contexto argentino, se realizó una retrotraducción (back-translation) del instrumento original en alemán. Una investigadora bilingüe tradujo en forma independiente los ítems del instrumento original del alemán al español. Luego, tres investigadores argentinos revisaron los ítems para corregir detalles mínimos de escritura y fraseo. Posteriormente, un quinto colaborador, también bilingüe, que no había tenido contacto con los ítems originales o traducidos, tradujo nuevamente los ítems del español al alemán. Finalmente, se compararon los ítems originales del HSCL-11 y el resultado de la traducción cruzada (back-translation) sin encontrar diferencias sustanciales.

Los participantes fueron convocados para participar del estudio a través de servicios de mensajería y distintas redes sociales. En la invitación enviada a los potenciales participantes se incluía un enlace que los dirigía a un sitio web especializado en la recolección de información para investigaciones (SurveyMonkey®). Allí se especificaba el único criterio de exclusión (tener menos de 18 años), también se informaba que la participación era voluntaria y anónima. Una vez que los participantes daban su consentimiento, completaban el HSCL-11, el PHQ-9, el GAD-7 y el WHO-5 (ver materiales). Al finalizar las escalas, se presentaron preguntas sociodemográficas y también algunas preguntas clínicas (las mismas se describen en la Tabla 1).

\section{Estrategias de análisis de datos}

Todos los análisis de este trabajo se realizaron en el software libre R (R Core Team, 2020). Específicamente, se utilizaron los paquetes psych (Revelle, 2019), Hmisc (Harrell, 2020) y lavaan (Rosseel, 2012). En el Apéndice 2 del trabajo se presenta el código completo de los análisis realizados y sus resultados, usando el paquete rmarkdown (Allaire et al., 2020).

Para estudiar la confiabilidad del instru- 
Tabla 1

Preguntas sociodemográficas y sus respectivas opciones de respuesta.

1. Lugar de residencia

$\begin{array}{ll}\text { a. Capital Federal } & \text { j. Formosa } \\ \text { b. Gran Buenos Aires } & \text { k. Jujuy } \\ \text { c. Provincia de Buenos Aires } & \text { 1. La Pampa } \\ \text { d. Catamarca } & \text { m. La Rioja } \\ \text { e. Chaco } & \text { n. Mendoza } \\ \text { f. Chubut } & \text { ñ. Misiones } \\ \text { g. Córdoba } & \text { o. Neuquén } \\ \text { h. Corrientes } & \text { p. Río Negro } \\ \text { i. Entre Ríos } & \text { q. Salta }\end{array}$

r. San Juan

s. San Luis

t. Santa Cruz

u. Santa Fe

v. Santiago del Estero

w. Tierra del Fuego, Antárti-

da e Islas del Atlántico Sur

x. Tucumán

y. Otro

2.Edad

3.Género
a. Femenino
b. Masculino
c. Otro

4. Nivel educativo avanzado
a. Primario incompleto
d. Secundario completo
g. Universitario incompleto
b. Primario completo
e. Terciario incompleto
h. Universitario completo
c. Secundario incompleto
f. Terciario completo

\section{Estado civil}
a. Soltero
b. Casado/en pareja
c. Separado/divorciado
d. Viudo

6. ¿Realizaste algún tratamiento psicológico o psiquiátrico durante el último año?
a. Sí
b. No

7. Si realizaste tratamiento psicológico o psiquiátrico, ¿cuál fueles el motivo principal?

a. Mejorar aspectos de algún área de mi vida (laboral, social, etc.)

b. Crisis vital (pérdida de algún ser querido, separación, despido o cambio de trabajo, etc.)

c. Malestar psicológico intenso (sentimientos de angustia, depresión, ansiedad, fobias, etc.)

d. Otro (especifique)

mento se utilizaron medidas de consistencia interna y homogeneidad de ítems. Así, se evaluó la consistencia interna del HSCL-11 calculando el coeficiente alfa de Cronbach y el coeficiente alfa ordinal. Si bien el alfa de Cronbach es la medida de consistencia interna más utilizada en la literatura, diversos estudios sugieren que no es la mejor estrategia cuando los ítems tienen una escala Likert con menos de siete opciones de respuesta (Freiberg-Hoffmann, Stover, de la Iglesia, \& Fernández-Liporace, 2013; Gadermann, Guhn, \& Zumbo, 2012). Por eso, en dichos casos se recomienda la utilización del alfa ordinal, una medida basada en correlaciones policóricas (Elosua-Oliden \& Zumbo, 2008; Zumbo, Gadermann, $\&$ Zeisser, 2007). El estado del arte sugiere que un valor aceptable para el coeficiente alfa oscila entre .70 y .90 . Valores más bajos representan una consistencia interna cuestionable, mientras que valores más altos implicarían que algunos ítems fueran redundantes (representando la misma pregunta con diferente formato; Tavakol \& Dennick, 2011). Por otro lado, para evaluar la homogeneidad de los ítems, se analizaron las correlaciones ítem-total corregidas (es decir, correlación promedio de cada ítem con el resto de los ítems de 
la escala). En este caso, se sugieren valores de correlación ítem-total en el rango de .30 a .80 (Rattray \& Jones, 2007). Correlaciones inferiores a 30 implican una escasa homogeneidad del ítem con el resto de los reactivos, mientras correlaciones más fuertes que .80 señalan que el ítem sería repetitivo.

Por su parte, para el estudio de la validez del instrumento se evaluó: la validez de constructo, la validez concurrente y la validez de criterio.

Resulta preciso señalar que la validez de constructo se estudió mediante un análisis factorial confirmatorio (AFC) utilizando el estimador diagonally weighted least squares (DWLS). Este estimador es una alternativa respecto de la máxima verosimilitud, que ha presentado superioridad al estudiar ítems que presentan un nivel de medición ordinal (Li, 2016; Mîndrilă, 2010). Para el establecimiento de la cantidad mínima necesaria de participantes, se tuvo en cuenta la cantidad de parámetros a estimar en el modelo del AFC. Debido a que el HSCL-11 es un instrumento unidimensional integrado por 11 ítems, la cantidad de parámetros a estimar en un AFC sería de 22 (11 cargas factoriales y 11 términos de error). Así, estableciendo un criterio de 15 sujetos por parámetro, se fijó un mínimo de 330 participantes para el estudio. Para la evaluación del ajuste del modelo en el AFC se utilizaron diversas medidas de bondad de ajuste tales como comparative fit index (CFI), Tucker-Lewis index (TLI), root mean square error of approximation (RMSEA) y standardized root mean square residual (SRMR). Como indicadores de buen ajuste del modelo, la literatura suele recomendar valores de CFI y TLI superiores a .95 y valores de SRMR y RMSEA inferiores a .08 (Schumacker \& Lomax, 2004). Por otra parte, para los modelos de AFC se consideran cargas de regresión aceptables aquellas iguales o por encima de .40 (Stover, Uriel, \& Fernandez-Liporace, 2012).
En segundo lugar, el estudio de la validez concurrente del HSCL-11 se realizó mediante correlaciones producto-momento de Pearson entre la escala y otras medidas de severidad psicopatológica. Particularmente se analizaron las correlaciones del instrumento con el PHQ-9 (medida de depresión), el GAD-7 (medida de ansiedad) y el WHO-5 (medida de bienestar psicológico). En este caso, las correlaciones deberían oscilar en el rango entre .50 y .85, indicando asociación, pero, a su vez, independencia de los constructos (Rial-Boubeta, Varela-Mallou, Abalo-Piñeiro, \& Lévy-Mangin, 2006).

Por último, la validez del criterio se evaluó mediante la comparación de los puntajes de HSCL-11 en sujetos que hayan o no realizado psicoterapia durante el último año, utilizando pruebas $t$ de Student de comparación de medias para muestras independientes. Frente a la presunción de que las personas que solicitan psicoterapia corresponderían a una población clínica con mayores niveles de severidad que la población no clínica (Jacobson \& Truax, 1991), se hipotetizó que aquellos sujetos que hubieran realizado terapia en el último año presentarían mayores puntajes de HSCL-11, en comparación con aquellos que no hubieran requerido tratamiento.

El código completo de los análisis realizados y sus resultados, presentados mediante el paquete rmarkdown, se encuentran publicados en la página del open science framework (subido el 25/01/2021 y hecho público el 18/03/2021, luego de la revisión anónima de pares), en el siguiente link: https://osf.io/mb5e7

\section{Resultados \\ Análisis descriptivos (Muestra 1)}

En la Tabla 2 se presentan los análisis descriptivos de los ítems del HSCL-11. Asimismo, 
en la Tabla 3 se reportan las medias y desvío estándar de los puntajes totales de todos los instrumentos utilizados en el estudio.

\section{Confiabilidad}

Consistencia interna. El HSCL-11 presentó un coeficiente alfa de Cronbach de .81. Considerando que los ítems del instrumento tienen una naturaleza ordinal y únicamente cuatro categorías de respuesta, además del coeficiente alfa de Cronbach se calculó también el coeficiente alfa ordinal, una medida de consistencia interna menos difundida, pero considerada más adecuada para este tipo de reactivos (Elosua-Oliden \& Zumbo, 2008; Zumbo et al., 2007). El alfa ordinal del HSCL-11 en este caso fue de .87 .
Homogeneidad de ítems. Las correlaciones ítem-total corregidas de los reactivos del HSCL11 se presentan en la Tabla 2. Como se observa en dicha tabla, únicamente el ítem 10 (p. ej., Pensar en quitarte la vida) presentó una correlación ítem-total corregida por debajo de .30. Por otra parte, ningún ítem obtuvo correlaciones ítem-total corregidas por encima de .80 .

\section{Validez de constructo}

El modelo testeado en el análisis factorial confirmatorio se ilustra en la Figura 1. Los resultados del mismo se presentan a su vez en la Tabla 4. Al evaluar las cargas factoriales estandarizadas del modelo, únicamente el ítem 10 (p. ej., Pensar en quitarte la vida), presentó cargas por debajo de 40. En cuanto a las medidas de bondad de ajus-

Tabla 2

Análisis descriptos y correlaciones ítem-total corregidas de los ítems del HSCL-11.

\begin{tabular}{|c|c|c|c|c|}
\hline Ítem & Media & $\begin{array}{c}\text { Desvío } \\
\text { estándar }\end{array}$ & $\begin{array}{c}r \text { ítem-total } \\
\text { corregida }\end{array}$ & Rango \\
\hline 1) Miedos & 1.92 & .84 & .57 & {$[1.4]$} \\
\hline 2) Nerviosismo & 2.39 & .86 & .56 & {$[1.4]$} \\
\hline 3) Sentirte tenso/a & 2.40 & .89 & .56 & {$[1.4]$} \\
\hline 4) Ataques de pánico & 1.18 & .49 & .41 & {$[1.4]$} \\
\hline 5) Problemas para dormir & 2.05 & .99 & .35 & {$[1.4]$} \\
\hline 6) Falta de esperanza en el futuro & 2.08 & .88 & .45 & {$[1.4]$} \\
\hline 7) Tristeza & 2.12 & .85 & .64 & {$[1.4]$} \\
\hline 8) Sentirte solo/a & 1.63 & .83 & .53 & {$[1.4]$} \\
\hline 9) No tener interés por nada & 1.69 & .90 & .42 & {$[1.4]$} \\
\hline 10) Pensar en quitarte la vida & 1.06 & .30 & .24 & {$[1.4]$} \\
\hline 11) Sentirte un/a inútil & 1.47 & .77 & .48 & {$[1.4]$} \\
\hline
\end{tabular}

Nota. Los ítems se contestan en una escala de 1 a 4 . La interpretación de los puntajes en la escala de respuesta se distribuye de la siguiente manera: $1=$ Nada $; 2=$ Poco $3=$ Bastante $4=$ Mucho.

Tabla 3

Análisis descriptos de los puntajes totales de los instrumentos utilizados en el estudio.

\begin{tabular}{lccc}
\hline Escalas & Media & Desvío estándar & Rango \\
\hline HSCL-11 & 1.82 & 0.47 & {$[1,3.45]$} \\
PHQ-9 & 0.56 & 0.46 & {$[0,2.56]$} \\
GAD-7 & 0.98 & 0.64 & {$[0,2.86]$} \\
WHO-5 & 2.32 & 1.03 & {$[0,4.80]$} \\
\hline
\end{tabular}


te se observaron un CFI de .97 y un TLI de .96 . El SRMR del modelo fue de .78, mientras que el RMSEA se ubicó en .05, IC 90\% [.04, .07].

\section{Validez externa}

Validez concurrente. Las correlaciones de Pearson producto-momento mostraron asociaciones directas significativas del HSCL-11, moderadas a fuertes, con el PHQ-9 (medida de depresión; $r=$ $.73, p<.001$ ) y el GAD-7 (medida de ansiedad; $r$ $=.75, p<.001)$. A su vez, se observó una correlación significativa inversa, moderadas a fuerte, del HSCL-11 con el WHO-5 (medida de bienestar; $r$ $=-.65, p<.001)$.

Validez de criterio. La prueba $t$ de Student para comparación de medias de muestras independientes demostró diferencias estadísticamente significativas en los niveles de HSCL-11, al comparar a los participantes que habían solicitado y que no habían solicitado realizar un tratamiento psico- terapéutico en el último año; $t_{(255)}=4.27,95 \%$ IC de la diferencia de medias $[.12, .33], p=.03$. Los pacientes que habían solicitado psicoterapia en el último año presentaron mayor puntaje en el HSCL-11 (Media $[\mathrm{M}]=1.95, \mathrm{DE}=.48)$, que los pacientes que no lo hicieron $(\mathrm{M}=1.73, \mathrm{DE}=.41)$.

\section{Discusión}

El objetivo de este trabajo ha sido presentar la adaptación al contexto argentino del Hopkins Symptoms Checklist (HSCL-11; Lutz et al., 2006) y explorar sus propiedades psicométricas. Los resultados de los análisis realizados mostraron evidencias de confiabilidad (consistencia interna y homogeneidad de ítems) y validez (validez de constructo, validez concurrente, validez de criterio) de la versión argentina del instrumento.

En cuanto a la consistencia interna, como primera medida de confiabilidad del instrumento, tanto el coeficiente alfa de Cronbach (medida de confiabilidad más difundida; $\alpha=.81$ ) como el

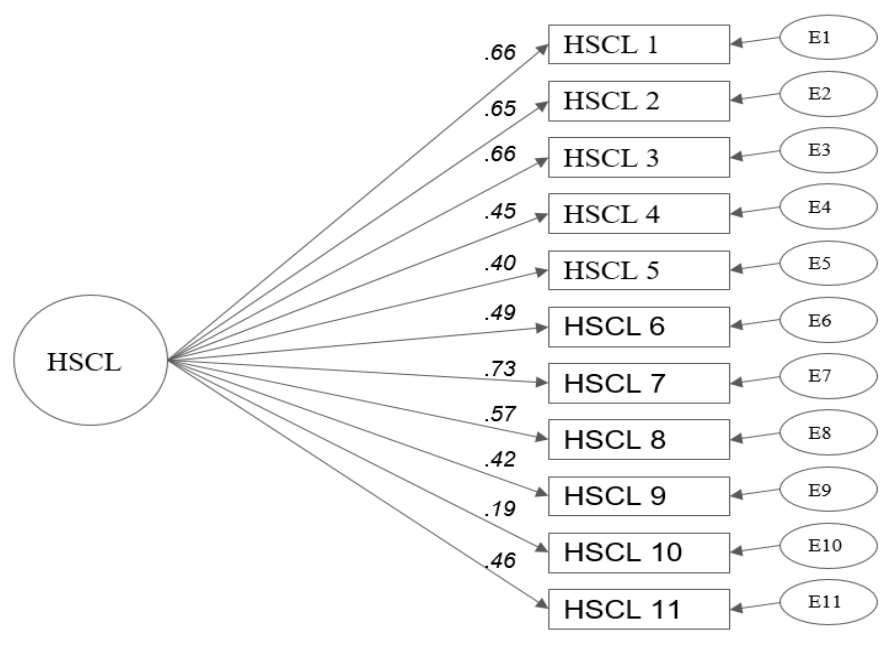

Figura 1

Parámetros estimados estandarizados del HSCL-11. 
Tabla 4

Cargas factoriales estandarizadas de los ítems del HSCL-11 en el modelo factorial confirmatorio.

\begin{tabular}{lccrc}
\hline Ítems & Parámetro & Error Estándar & $\boldsymbol{z}$ & $\boldsymbol{p}$ \\
\hline Item1 & .66 & .04 & 16.42 & $<.001$ \\
Item2 & .65 & .04 & 15.40 & $<.001$ \\
Item3 & .66 & .04 & 15.56 & $<.001$ \\
Item4 & .45 & .05 & 8.42 & $<.001$ \\
Item5 & .40 & .05 & 7.78 & $<.001$ \\
Item6 & .49 & .05 & 9.91 & $<.001$ \\
Item7 & .73 & .03 & 21.48 & $<.001$ \\
Item8 & .57 & .05 & 12.36 & $<.001$ \\
Item9 & .42 & .06 & 7.19 & $<.001$ \\
Item10 & .19 & .07 & 2.66 & $<.001$ \\
Item11 & .46 & .05 & 9.28 & $<.001$ \\
\hline
\end{tabular}

coeficiente alfa ordinal (alternativa para variables ordinales; $\alpha$ ordinal $=.87$ ) se ubicaron dentro del rango de .70 y .90 . Esto implica que los ítems presentaron un nivel aceptable de consistencia interna sin que los reactivos fuesen redundantes (Tavakol \& Dennick, 2011).

Por otra parte, al evaluar la homogeneidad de los ítems, como otra medida de confiabilidad, todos los ítems - excepto uno- presentaron correlaciones ítem-total en el rango sugerido (Rattray \& Jones, 2007). Únicamente un reactivo (ítem 10: Pensar en quitarte la vida) presentó una escasa homogeneidad con el resto de los ítems (es decir, correlaciones por debajo de .30). Como se puede observar en la Tabla 2, dicho ítem presentó niveles muy bajos de variabilidad en sus respuestas, siendo el desvío estándar promedio de los otros ítems $(\mathrm{DE}=.83)$ casi tres veces mayor que el observado en este reactivo (DE = .30). Esta escasa variabilidad podría explicar las bajas correlaciones del ítem con los otros reactivos (Goodwin \& Leech, 2006). Por otra parte, todos los ítems presentaron correlaciones ítem-total por debajo de .80, sugiriendo nuevamente que los ítems no eran repetitivos ni redundantes (Rattray \& Jones, 2007).

El análisis factorial confirmatorio realizado mostró evidencias de validez de constructo del instrumento. La estructura unidimensional mode- lizada presentó medidas de bondad de ajuste por encima de las sugeridas para estos casos. Tanto el CFI (.97) como el TLI (.96) se ubicaron por encima de .95 (Schumacker \& Lomax, 2004). A su vez, el SRMR (.078) y el RMSEA (.05, IC $90 \%[.04, .07])$ se encontraron por debajo de .08 (Schumacker \& Lomax, 2004). No obstante, cabe destacar que un ítem (nuevamente el ítem 10, correspondiente a Pensar en quitarte la vida) no cumplió con el criterio de presentar cargas de regresión por encima de .40 (Stover et al., 2012). Al igual que en el caso de la homogeneidad, es posible que las bajas cargas de regresión de este ítem se expliquen por la escasa variabilidad del reactivo (Goodwin \& Leech, 2006).

Las correlaciones de Pearson del HSCL-11 con medidas de depresión ( $r$ con PHQ-9 $=.73$ ), ansiedad ( $r$ con GAD-7 $=.75$ ) y bienestar psicológico ( $r$ con WHO-5 = -.65), sugieren evidencia de validez concurrente del instrumento. Al ubicarse todas las correlaciones en el rango de .50 .85 , esto implica que los instrumentos se ven asociados entre sí, pero a su vez exploran constructos independientes (Rial-Boubeta et al., 2006).

Finalmente, para obtener evidencias respecto de la validez del criterio del HSCL-11, se realizaron pruebas $t$, analizando si existían diferencias significativas en sus puntajes entre participantes que habían realizado psicoterapia en el último 
año y participantes que no se habían involucrado en un tratamiento psicoterapéutico en ese periodo de tiempo. De esta manera, se buscó establecer si los puntajes del HSCL-11 permitían diferenciar una población clínica de una población no clínica (Jacobson \& Truax, 1991). Los resultados de la prueba $t$ arrojaron evidencias de validez de criterio del HSCL-11, presentando diferencias estadísticamente significativas entre los dos grupos, y el conjunto de pacientes de psicoterapia mostró mayores niveles de severidad según los puntajes del instrumento.

Estos resultados en su conjunto presentan al HSCL-11, en su versión argentina, como un instrumento con evidencias de confiabilidad $\mathrm{y}$ validez que puede representar un recurso valioso para evaluar sintomatología psicológica y, especialmente por su brevedad, para el monitoreo de pacientes sesión a sesión durante tratamientos psicoterapéuticos.

Cabe aclarar que este estudio presenta una serie de limitaciones que deberán ser abordadas en futuras investigaciones. En primer lugar, la exploración de la validez concurrente se realizó sobre la base de correlaciones del HSCL-11 con otras medidas, completadas también mediante autoinformes de los participantes. Es posible que el hecho de que todas las medidas sean completadas por el mismo sujeto "infle" las correlaciones, debido a la existencia de una fuente común. Futuros trabajos se beneficiarían de utilizar y triangular medidas de sintomatología completadas por otras fuentes (observadores externos, conocidos, terapeutas, etc.) para explorar la validez concurrente del HSCL-11. Además, en este estudio no se analizó la validez de contenido del instrumento mediante la evaluación del grado de acuerdo de jueces expertos, respecto de la relevancia de los ítems utilizados. Por lo tanto, sería útil estudiar esta dimensión de validez en la versión argentina del HSCL-11 en futuros trabajos. Por otra parte, en este estudio tampoco se utilizó un método estructurado de diagnóstico psiquiátrico que permitiera diferenciar entre una población patológica y una estándar, lo que impidió obtener información relevante para el uso clínico del instrumento, como identificación de puntos de corte clínico desarrollados mediante análisis de curvas ROC. En el futuro deberían realizarse análisis diferenciales del HSCL-11 en participantes que presenten diagnósticos psicopatológicos (por ejemplo, trastornos de ansiedad o trastornos depresivos) y participantes que integren una población regular o estándar (es decir, no patológica), usando procedimientos estructurados de evaluación para determinar empíricamente su diferencia y establecer puntos de corte clínicos. A su vez, algunas características demográficas de la muestra, como los altos porcentajes de participantes con estudios terciarios/universitarios, presentan limitaciones respecto de la capacidad de generalización de los resultados a la población. Nuevas investigaciones sobre el HSCL-11 deberían utilizar muestreos probabilísticos o por cuotas, que permitan una distribución más representativa de la población en la muestra de estudio y, por ende, mayor validez externa de las inferencias psicométricas. Por otra parte, la muestra de este trabajo fue recolectada exclusivamente mediante redes sociales, utilizando una estrategia de bola de nieve. Al no contar con datos de participantes convocados mediante otras estrategias, resulta imposible determinar si la estrategia seleccionada pudo haber sesgado los resultados obtenidos en una dirección específica. A futuro sería relevante analizar las propiedades del HSCL-11 en Argentina usando muestras recolectadas con distintas estrategias para observar si el método de muestreo influye en sus propiedades psicométricas (por ejemplo, testeando la invarianza factorial en el análisis confirmatorio entre participantes convocados vía redes sociales en comparación con participantes convocados por 
otros medios).

Finalmente, para estudiar la capacidad del HSCL-11 como instrumento de monitoreo de terapias psicológicas, sería necesario estudiarlo en una muestra de pacientes, en donde se tomen medidas repetidas sesión a sesión, o incluso con medidas ecológicas intensivas, para poder estudiar su sensibilidad al cambio, así como también su capacidad de implementación dentro de un sistema de feedback para terapeutas. Más allá de estas limitaciones, los resultados del artículo presentan al HSCL-11 en su versión argentina como un instrumento con evidencias de confiabilidad y validez, que además tiene el potencial de convertirse en un recurso importante para la evaluación y el tratamiento de patologías mentales en el país.

\section{Referencias}

Abuín, M. R., \& de Rivera, L. (2014). La medición de síntomas psicológicos y psicosomáticos: El Listado de Síntomas Breve (LSB-50). Clínica y Salud, 25(2), 131-141. doi: 10.1016/j.clysa.2014.06.001

Allaire, J. J., Xie, Y., McPherson, J., Luraschi, J., Ushey, K., Atkins, A., ... \& Iannone, R. (2020). Rmarkdown: Dynamic Documents for R (2.6). [Software de cómputo]. Recuperado de https://rmarkdown.rstudio.com

Areas, M., Roussos, A., Hirsch, H., Hirsch, P., Becerra, P., \& Gómez-Penedo, J. M. (2018). Evaluación de un dispositivo de investigación orientada por la práctica para el desarrollo de un sistema de feedback en psicoterapia. Revista Argentina de Clínica Psicológica, 27(2), 229-249. doi: 10.24205/03276716.2018.1064

Barros, J., Morales, S., García, A., Echávarri, O., Fischman, R., Szmulewicz, M., ... \& Tomicic, A. (2020). Recognizing states of psychological vulnerability to suicidal behavior: A Bayesian network of artificial intelligence applied to a clinical sample. BMC Psychiatry, 20, 138. doi: 10.1186/s12888-020-02535-X

Boswell, J. F., Constantino, M. J., Kraus, D. R., Bugatti, M.,
\& Oswald, J. M. (2015). The expanding relevance of routinely collected outcome data for mental health care decision making. Administration and Policy in Mental Health and Mental Health Services Research, 43, 482-491. doi: 10.1007/s10488-015-0649-6

Botella i García del Cid, L. (2008). Resultado y proceso en Psicoterapia Cognitivo-Constructivista Integradora. Apuntes de Psicología, 26(2), 229-241. Recuperado de http://www.apuntesdepsicologia.es/index.php/revista/index

Casullo, M. (1999/2008). El inventario de síntomas SCL90-R de L. Derogatis. Documento de Trabajo. Facultad de Psicología, UBA. Recuperado de http://www. fundacionforo.com/pdfs/inventariodesintomas.pdf

Chisholm, D., Sweeny, K., Sheehan, P., Rasmussen, B., Smit, F., Cuijpers, P., \& Saxena, S. (2016). Scaling-up treatment of depression and anxiety: A global return on investment analysis. The Lancet Psychiatry, 3(5), 415-424. doi: 10.1016/S2215-0366(16)30024-4

Constantino, M. J., Coyne, A. E., \& Muir, H. J. (2020). Evidence-based therapist responsivity to disruptive clinical process. Cognitive and Behavioral Practice, 27(4), 405-416. doi: 10.1016/j.cbpra.2020.01.003

Cornelio, C., \& Contreras, A. (2020). WHO-5 Index: Validez, confiabilidad y aplicaciones de una escala para evaluar el bienestar subjetivo en salud laboral. Documento técnico. Ministerio de Trabajo, Empleo y Seguridad Social. Recuperado de https://www.argentina.gob.ar/srt/observatorio-srt

De la Iglesia, G., Stover, J. B., Castro-Solano, A., \& Fernández-Liporace, M. (2015). Validez de constructo del LSB-50 en adultos argentinos: Validación cruzada e invarianza factorial. Acción Psicológica, 12(2), 43-58. doi: 10.5944/ap.12.2.15327

Delgadillo, J., \& Lutz, W. (2020). A development pathway towards precision mental health care. JAMA Psychiatry, 77(9), 889-890. doi: 10.1001/jamapsychiatry. 2020.1048

Derogatis, L. R. (1983). SCL-90-R: Administration, Scoring and Procedures Manual II for the R (evised) Version of the SCL-90. Baltimore, MD: John Hopkins Uni- 
versity School of Medicine, Clinical Psychometric Research.

Elosua-Oliden, P., \& Zumbo, B. D. (2008). Coeficientes de fiabilidad para escalas de respuesta categórica ordenada. Psicothema, 20(4), 896-901. Recuperado de http://www.psicothema.com

Emmelkamp, P. M. G., David, D., Beckers, T., Muris, P., Cuijpers, P., Lutz, W., ... \& Vervliet, B. (2014). Advancing psychotherapy and evidence-based psychological interventions. International Journal of Methods in Psychiatric Research, 23(S1), 58-91. doi: 10.1002/mpr.1411

Feixas, G., Evans, C., Trujillo, A., Saúl, L. A., Botella, L., Corbella, S., ... \& López-González, M. A. (2012). La versión española del CORE-OM: Clinical Outcomes in Routine Evaluation - Outcome Measure. Revista de Psicoterapia, 23(89), 109-135. Recuperado de https://ojs.revistadepsicoterapia.com/index.php

Fernández-Álvarez, J., Fernández-Álvarez, H., \& Castonguay, L. G. (2018). Resumiendo los nuevos esfuerzos para integrar la práctica y la investigación desde la perspectiva de la investigación orientada por la práctica. Revista Argentina de Clínica Psicológica, 27(2), 353-372. doi: 10.24205/03276716.2018.1070

Fernández-Álvarez, H., Hirsch, H., Maristany, M., \& Torrente, F. (2005). Propiedades psicométricas del OQ45.2 en la Argentina: Un estudio piloto. Poster presentado en el $4^{\circ}$ Congreso Mundial de Psicoterapia, Buenos Aires.

Freiberg-Hoffmann, A., Stover, J. B., de la Iglesia, G., \& Fernández-Liporace, M. (2013). Correlaciones policóricas y tetracóricas en estudios factoriales exploratorios y confirmatorios. Ciencias Psicológicas, 7(2), 151-164. Recuperado de https://revistas.ucu.edu.uy/ index.php/cienciaspsicologicas

Gadermann, A. M., Guhn, M., \& Zumbo, B. D. (2012). Estimating ordinal reliability for likert-type and ordinal item response data: A conceptual, empirical, and practical guide. Practical Assessment, Research and Evaluation, 17, Art. 3. Recuperado de https://scholarworks.umass.edu/pare
García-Campayo, J., Zamorano, E., Ruiz, M. A., Pardo, A., Pérez-Páramo, M., López-Gómez, V., ... \& Rejas, J. (2010). Cultural adaptation into Spanish of the generalized anxiety disorder-7 (GAD-7) scale as a screening tool. Health and Quality of Life Outcomes, 8, Art. 8. doi: 10.1186/1477-7525-8-8

Gómez-Penedo, J. M., Zilcha-Mano, S., \& Roussos, A. (2019). Interpersonal profiles in emotional disorders predict the importance of alliance negotiation for early treatment outcome. Journal of Consulting and Clinical Psychology, 87(7), 617-628. doi: 10.1037/ ccp0000417

Goodwin, L. D., \& Leech, N. L. (2006). Understanding correlation: Factors that affect the size of $r$. Journal of Experimental Education, 74(3), 249-266. doi: 10.3200/JEXE.74.3.249-266

Harrell, F. E. Jr. (2020). Hmisc: Harrell Miscellaneous. R package version 4.4-0. [Rstudio]. Recuperado de https://CRAN.R-project.org/package=Hmisc

Jacobson, N. S., \& Truax, P. (1991). Clinical significance: A statistical approach to defining meaningful change in psychotherapy research. Journal of Consulting and Clinical Psychology, 59(1), 12-19. doi: 10.1037/0022-006X.59.1.12

Kroenke, K., Spitzer, R. L., \& Williams, J. B. (2001). The PHQ-9: Validity of a brief depression severity measure. Journal of General Internal Medicine, 16(9), 606613. doi: 10.1046/j.1525-1497.2001.016009606.x

Lambert, M. J. (2013). Outcome in psychotherapy: The past and important advances. Psychotherapy, 50(1), 4251. doi: 10.1037/a0030682

Lambert, M., Hansen, N., Umpress, V., Lunnen, K., Okiiski, J., Burlingame, G., \& Reisinger, C. W. (1996). Administration and Scoring Manual for the Outcome Questionnarie (OQ45-2). Wilmington, DE: American Professional Credentialing.

Lara, C., Cruz, C., Vacarezza, A., Florenzano, R., \& Trapp, A. (2008). Análisis comparativo de dos instrumentos de evaluación clínica: OQ-45 e interRAI- Salud Mental. Revista Chilena de Neuro-psiquiatría, 46(3), 192-198. doi: 10.4067/S0717-92272008000300004 
Li, C. -H. (2016). The performance of ML, DWLS, and ULS estimation with robust corrections in structural equation models with ordinal variables. Psychological Methods, 21(3), 369-387. doi: 10.1037/met0000093

Lucas-Carrasco, R. (2012). The WHO Quality Of Life (WHOQOL) Questionnaire: Spanish development and validation studies. Quality of Life Research, 21(1), 161-165. doi: 10.1007/s11136-011-9926-3

Lutz, W., Tholen, S., Schürch, E., \& Berking, M. (2006). Die Entwicklung, Validierung und Reliabilität von Kurzformen gängiger psychometrischer Instrumente zur Evaluation destherapeutischen Fortschritts in Psychotherapie und Psychiatrie. Diagnostica, 52(1), 11-25. doi: 10.1026/0012-1924.52.1.11

Lutz, W., de Jong, K., \& Rubel, J. (2015). Patient-focused and feedback research in psychotherapy: Where are we and where do we want to go? Psychotherapy Research, 25(6), 625-632. doi: 10.1080/10503307.2015.1079661

Lutz, W., Rubel, J. A., Schwartz, B., Schilling, V., \& Deisenhofer, A. (2019). Towards integrating personalized feedback research into clinical practice: Development of the Trier Treatment Navigator (TTN). Behaviour Research and Therapy, 120, 103438. doi: 10.1016/j.brat.2019.103438

Mîndrilă, D. (2010). Maximum Likelihood (ML) and Diagonally Weighted Least Squares (DWLS) estimation procedures: A comparison of estimation bias with ordinal and multivariate non-normal data. International Journal of Digital Society, 1(1), 60-66. doi: 10.20533/ijds.2040.2570.2010.0010

Organización Mundial de la Salud. (1998). Wellbeing measure in primary health care/The Depcare Project. Copenhagen, Denmark: WHO Regional Office for Europe.

Paz, C., Mascialino, G., \& Evans, C. (2020). Exploration of the psychometric properties of the Clinical Outcomes in Routine Evaluation-Outcome Measure in Ecuador. BMC Psychology, 8(1), Art. 94. doi: 10.1186/s40359-020-00443-Z

R Core Team. (2020). R: A language and environment for statistical computing. [Software de cómputo]. R Foundation for Statistical Computing, Vienna, Austria. Recuperado de https://www.R-project.org

Rattray, J., \& Jones, M. C. (2007). Essential elements of questionnaire design and development. Journal of Clinical Nursing, 16(2), 234-243. doi: 10.1111/j.1365-2702.2006.01573.x

Revelle, W. (2019). psych: Procedures for personality and psychological research (1.9.12). [Software de cómputo]. Recuperado de https://CRAN.R-project.org

Rial-Boubeta, A., Varela-Mallou, J., Abalo-Piñeiro, J., \& Lévy-Mangin, J. P. (2006). El análisis factorial confirmatorio. En J. P. Lévy-Mangin \& J. Varela-Mallou (Eds.), Modelización con estructuras de covarianzas en ciencias sociales (pp. 119-154). Coruña, España: Netbiblo.

Rosseel, Y. (2012). lavaan: An R package for structural equation modeling. Journal of Statistical Software, 48(2), 1-36. doi: 10.18637/jss.v048.i02

Roussos, A. J., Gómez-Penedo, J. M., \& Muiños, R. (2018). A time-series analysis of therapeutic alliance, interventions, and client's clinical status in an evidence-based single-case study: Evidence for establishing change mechanisms in psychotherapy. Psychotherapy Research, 28(1), 137-149. doi: 10.1080/10503307.2016.1174346

Schumacker, R. E., \& Lomax, R. G. (2004). A beginner's guide to structural equation modeling ( $4^{\mathrm{a}}$ ed.). New York, NY:. Psychology Press. doi: 10.4324/9781410610904

Sosa-Torralba, J. E., Romero-Mendoza, M. P., Medina-Mora-Icaza, M. E., Méndez-Ríos, E., \& Blum-Grynberg, B. (2020). Estructura dimensional de la Escala Clinical Outcomes in Routine Evaluation-Outcome Measure (CORE-OM) en universitarios mexicanos. Revista Evaluar, 20(1), 49-66. Recuperado de https:// revistas.unc.edu.ar/index.php/revaluar

Spitzer, R. L., Kroenke, K., Williams, J. B., \& Löwe, B. (2006). A brief measure for assessing generalized anxiety disorder: the GAD-7. Archives of Internal Medicine, 166(10), 1092-1097. doi: 10.1001/archin- 
te.166.10.1092

Stover, J. B., Uriel, F., \& Fernández-Liporace, M. (2012). Inventario de estrategias de aprendizaje y estudio: Análisis psicométricos de una versión abreviada. Revista Argentina de Ciencias Del Comportamiento, 4(3), 4-12. Recuperado de https://revistas.unc.edu. ar/index.php/racc

Tavakol, M., \& Dennick, R. (2011). Making sense of Cronbach's alpha. International Journal of Medical Education, 2, 53-55. doi: 10.5116/ijme.4dfb.8dfd

Urtasun, M., Daray, F. M., Teti., G. L., Coppolillo, F., Herlax, G., Saba, G., ... \& Irazola, V. (2019). Validation and calibration of the Patient Health Questionnaire (PHQ-9) in Argentina. BMC Psychiatry, 19(1), Art. 291. doi: 10.1186/s12888-019-2262-9

Zilcha-Mano, S., \& Errázuriz, P. (2015). One size does not fit all: Examining heterogeneity and identifying moderators of the alliance-outcome association. Journal of Counseling Psychology, 62(4), 579-591. doi: 10.1037/cou0000103

Zumbo, B. D., Gadermann, A. M., \& Zeisser, C. (2007). Ordinal versions of coefficients alpha and theta for Likert rating scales. Journal of Modern Applied Statistical Methods, 6(1), 21-29. doi: 10.22237/ jmasm/1177992180 